\title{
DEMOGRAPHIC AND CLINICAL PROFILES AND MORTALITY OF ALUMINIUM PHOSPHIDE POISONING CASES IN KHYBER PAKHTUNKHWA PROVINCE, PAKISTAN
}

\author{
Sakhi Jan', (DHamid Shahzad1, (DHassan Naveed', (DAtta Ullah', (D)Muhammad Bilal', \\ Mahnoor Asad ${ }^{2}$ \\ ${ }^{1}$ Emergency Department, Lady Reading Hospital, ${ }^{2}$ Khyber Medical College, Peshawar, Pakistan
}

\begin{abstract}
Background: The prevalence of aluminium phosphide (AIP) poisoning is on the rise in Asian countries. The objectives of our study were to determine the distribution of AIP poisoning by sex, age groups \& district and its presentation by blood pressure category and oxygen saturation category and its mortality in Khyber Pakhtunkhwa Province, Pakistan.

Materials \& Methods: This cross-sectional study was conducted at Emergency Department of Lady Reading Hospital, Peshawar, Pakistan from June 1, 2020 to September 30, 2020. Fifty eight cases of AIP registered during 2019 were included. The diagnosis was made by history and clinical suspicion. All these cases were managed by standard management protocols. Sex, age groups, district, blood pressure category, oxygen saturation category and presence of mortality were variables; all being categorical were analyzed by count and percentage with $80 \%$ confidence interval.
\end{abstract}

Results: Out of 58 AIP poisoning cases, women 34 (58.62\%) were more than men 24 (41.38\%), with most cases $30(51.73 \%)$ in $10-19$ years age group. Most cases 17 (29.31\%) were from district Swabi, followed by district Peshawar 16 (27.58\%). Most cases presented in non-recordable blood pressure category 19 (32.76\%). Most cases $22(37.93 \%)$ presented in oxygen saturation category of $\geq 90 \%$. Out of 58 cases, mortality was $72.41 \%$ (42/58), involving more women 24/58 (41.38\%) than men 18/58 (31.03\%), with most cases $23(39.66 \%)$ in 10-19 years age group. Mortality was highest in non-recordable blood pressure category $19(32.76 \%)$. Mortality was highest $19(32.76 \%)$ in oxygen saturation category of below $90 \%$.

Conclusion: Aluminium phosphide (AIP) poisoning is an uncommon, but a dreadful public health problem in population of Khyber Pakhtunkhwa Province, Pakistan. It involves women more than men, with most cases in adolescent age group, and in non-recordable blood pressure category. AIP poisoning mortality is very high $72.41 \%$ in our population, involving more women than men, with most cases in adolescent age group. Mortality is highest in non-recordable blood pressure category and in oxygen saturation category of below $90 \%$.

KEY WORDS: Aluminium Phosphide; Poisoning; Mortality; Blood Pressure; Hypotension; Ingestion; Sex; Age Groups; Pakistan; Asia.

Cite as: Jan S, Shahzad H, Naveed H, Ullah A, Bilal M, Asad M. Demographic and clinical profiles and mortality of aluminium phosphide poisoning cases in Khyber Pakhtunkhwa Province, Pakistan. Gomal J Med Sci 2021 Jan-Mar; 19(1):11-8. https://doi.org/10.46903/gjms/19.01.931

\section{Corresponding Author:}

Dr. Hassan Naveed

Trainee Medical Officer

Emergency Department, Lady Reading Hospital

Peshawar, Pakistan

E-mail: hassaannaveed621@gmail.com

Date Submitted:

05-09-2020

Date Revised:

Date Accepted:
$12-11-2020$

$10-12-2020$

\section{INTRODUCTION}

1.1 Background: Each year, nearly 300,000 people die due to pesticide poisoning around the globe. The most familiar pesticides are organophosphates and phosphides. Among phosphides, aluminium phosphide (AIP), the active substance present in wheat pills, is vastly utilized in agricultural communities as a pesticide that kills rodents. ${ }^{1}$ The pill contains around $44 \%$ inert elements to avoid disintegration of the tablet, while the rest (about $56 \%$ ) is 
aluminium phosphide. The lethal dose of aluminium phosphide is between 0.15 and 0.5 grams $(0.0053$ and $0.0176 \mathrm{oz}$ ). Aluminium phosphide is available in the form of $3 \mathrm{gm}$ pellets (releasing $1 \mathrm{gm}$ phosphine gas) or $0.6 \mathrm{gm}$ pellets (releasing $0.2 \mathrm{gm}$ phosphine gas). ${ }^{2}$

The mechanism of action consists of the formation of phosphine gas as the end product that results in damage to mitochondria by the action of free radicals. It causes conformational changes in mitochondria and inhibits oxidative respiration up to $70 \% .^{3,4}$ Phosphine gas induces damage to cell membranes by inhibition of acetyl cholinesterase, inhibition of peroxidase and/ or inhibition of glycerol phosphate dehydrogenase. ${ }^{5}$ Multiple researches show that phosphides result in the formation Heinz bodies by denaturing hemoglobin, which causes intravascular hemolysis and methemoglobinemia. This leads to fatal complications such as gastritis, hepatic necrosis, disseminated intravascular coagulation, cardiac arrhythmia, metabolic acidosis, congestive heart failure, hypotensive shock and eventually multi-organ dysfunction syndrome. ${ }^{3}$ Hypotension was the commonest presenting complaint of wheat pill poisoning followed by vomiting. ${ }^{6}$

The management of aluminium phosphide poisoning mainly consists of supportive measures as there is no specific antidote. The cardinal supportive measures comprise of securing air way, optimizing blood pressure and to prevent hypoxia of the tissues. Severity of the intoxication can be observed by serial blood levels of phosphine (PH3) during the acute intoxication. ${ }^{7}$ Gastric lavage with potassium permanganate and mineral oil or coconut oil shows a decrease in mortality. ${ }^{8}$ Hypotensive shock as a manifestation of wheat pill poisoning is often recurrent and usually requires aggressive resuscitation via IV fluids guided by central venous pressure (CVP) and a pulmonary capillary wedge pressure (PCWP). As a consequence of fluids resistant hypotension, low dose dopamine (at 4-6 $\mu \mathrm{g} / \mathrm{kg} / \mathrm{min}$ ) should be administered to keep systolic blood pressure above $90 \mathrm{mmHg}$. IV hydrocortisone (200-400mg q 4- 6 hours) aids in dopamine dose reduction and prevents development of acute respiratory distress syndrome (ARDS) by inhibition of capillary leakage in the lungs. In addition, the renal route can be utilized to increase the excretion of phosphine by giving IV furosemide if the systolic BP is greater than $90 \mathrm{mmHg}$. IV sodium bicarbonate $(50-100 \mathrm{mEq}$ q 8 hours) should be used in order to achieve the target bicarbonate level of $18-20 \mathrm{mEq} / \mathrm{L}$. A small number of case studies have shown beneficial effects of digoxin in the management of refractory cardiac shock. ${ }^{9}$ In 12-24 hours of initial presentation of acute aluminium phosphide poisoning, cardiovascular issues such as hypotension and/ or arrhythmias have been the most common causes of morbidity. The next 24 hours are associated with liver failure as the usual cause of death. ${ }^{6}$ All patients with AIP poisoning require cardiac monitoring and electrolytes, such as potassium and magnesium replacement. Low magnesium might lead to Torsades de Pointes and ventricular fibrillation, which should be managed according to the ACLS guidelines. Although debatable, magnesium replacement is the only intervention linked to decreased mortality in AIP poisoning..$^{10}$ Magnesium replacement has also been shown to provide an anti-peroxidant effect. Chugh, et al. illustrated the effect of magnesium to prevent free radicals induced damage which is gradual and autonomous of phosphine elimination. ${ }^{11}$ Some case reports indicate development of arrhythmias such as the Brugada syndrome like toxic myocarditis in AIP poisoning. ${ }^{12}$ Heart failure is commonly associated with acute pulmonary edema. ${ }^{13}$ Hypotensive shock can be treated with ionotropic support, digoxin and trimetazidine. Trim etazidine helps to halt ventricular ectopic beats and bigeminy and conserves oxidative metabolism. ${ }^{14} \mathrm{~A}$ few case reports designate the effects of coconut oil in the acute management of AIP poisoning. ${ }^{8}$ Shahdina, et al. reported reduced absorption of phosphine gas as a major effect of coconut oil, which has been shown to be efficacious even after 6 hours of acute aluminum phosphide ingestion. Some animal studies revealed that hyperbaric oxygen therapy might result in an improvement of survival in animals exposed to aluminum phosphide. $\mathrm{N}$-acetylcysteine (NAC), being an antioxidant, reduces mortality in acute AIP poisoning. In a randomized controlled trial conducted in Iran, NAC lowered the period of hospitalization, need for intubation and ventilation. ${ }^{15}$ In general, AIP poisoning patients show poor prognosis with mortality nearly $70 \%$. Poor prognostic signs in AIP poisoning include, low Glasgow coma scale, shock, ECG changes, low prothrombin rate, hyperleukocytosis, use of mechanical ventilation, use of vasoactive drugs and high APACHE II (Acute Physiology and Chronic Health Evaluation II). ${ }^{16}$

From January 1997 to June 2003, 93 cases of aluminum phosphide exposures were reported to the National Poisons Information Service, London; 8 aluminium phosphide ingestions, 57 inhalational cases of phosphine gas, 3 topical exposures, 11 oral/ inhalational or oral/ topical and 14 with unknown exposure route. Among these cases, 17 were asymptomatic, 33 had respiratory symptoms, and 27 had gastrointestinal symptoms. Only one death of an 80 year old male was reported. However, majority of the cases were caused by accidental, comparatively restricted exposure in agricultural environments to phosphine gas. The incidences of self-induced AIP poisoning cases do not seem to be growing. ${ }^{17} \mathrm{~A}$ North Indian study reported it to be the most common way of committing suicide in 
the region. ${ }^{18,19}$ Among 559 cases of acute poisoning in India, $68 \%$ were as a result of AIP exposure, with $60 \%$ mortality. ${ }^{19}$ Gupta and Ahlawat have also validated this suicidal AIP poisoning epidemic. ${ }^{20}$ In the period between 1977 and 1987, barbiturates $(33.3 \%)$, organophosphates $(23.8 \%)$, and copper sulphate $(14.3 \%)$ were the most common suicidal agents and from 1987 to 1997 , they were substituted by organophosphates ( $45 \%)$ and AIP (26.5\%). Since 1992 , AIP has taken lead with as much as $80 \%$ of the total cases. The incidence of suicidal deaths grew from $10.9 \%$ (1987 to 1992) to $15.7 \%$ (1997 and 2002), with the highest occurrence of $18.2 \%$ (1992 to 1997), as AIP became available on the free market. Of all deadly toxins, both suicidal and accidental between 1987 and 1997, AIP attributed to $26.5 \%$. AIP proved to be the most common poison to commit suicide, accounting for $68.4 \%$ of total fatalities due to poisoning in 10 years (1992 to 2002). Another study carried out in Tehran, Iran concluded that there was a noteworthy rise $(p<0.05)$ in mortality due to phosphine poisoning in an 8-year period..$^{21}$ The rate of AIP poisonings in Iran has also been shown to be high; among 471 cases reported from 2000 to 2007,146 (31\%) were lethal, ${ }^{22}$ which is increasingly worrying considering the fact that AIP marketing is illegal in Iran. A study conducted in Saudi Arabia reported death rate in AIP poisonings was highest among children and a majority of them occurred during fumigation of households. In developed states such as the UK, however, AIP is available in the form of pills, but supply is regulated under the 1998 Pesticides Act to certified customers. ${ }^{17}$ AIP tablets are only rarely used in European countries to commit suicides and a small number of cases have been reported in the UK, Denmark, Germany and France. Demographic data for Pakistani population, however, is not available. ${ }^{6}$

1.2 Research Objectives (ROs): The objectives of our study were;

RO 1-3: To determine the distribution of aluminium phosphide poisoning by sex, age groups \& district in Khyber Pakhtunkhwa Province, Pakistan.

RO 4-5: To determine the presentation of aluminium phosphide poisoning by blood pressure and oxygen saturation in Khyber Pakhtunkhwa Province, Pakistan

RO 6-8: To determine the prevalence of aluminium phosphide poisoning mortality and its distribution by sex and age groups in Khyber Pakhtunkhwa Province, Pakistan.

RO 9-10: To determine the presentation of aluminium phosphide poisoning mortality by blood pressure and oxygen saturation in Khyber Pakhtunkhwa Province, Pakistan.

1.3 Significance: Having in hand the local data on demographic profile, clinical profile and mortality of aluminium phosphide poisoning cases in Khyber Pakhtunkhwa Province, Pakistan, we can formulate indigenous guidelines for diagnosis and treatment of such cases. This data base will help other researcher working on this title, and other titles on its association to socio-demographic factors.

\section{MATERIALS AND METHODS}

2.1 Design, Setting \& Duration: This cross-sectional study was conducted at the Emergency Department of Lady Reading Hospital, Peshawar, Pakistan from June 1, 2020 to September 30, 2020. This hospital is the oldest and largest teaching hospital of the Khyber Pakhtunkhwa province and has largest inflow of casualty patients. The data for the year 2019 was retrospectively observed for AIP cases after permission was sought from relevant authorities.

2.2 Sample Selection \& Conduct of Procedure: Fifty eight (58) cases of AIP registered during the year 2019 were included. There were no exclusion criteria. The diagnosis was made by history and clinical suspicion. All these AIP poisoning cases were managed by standard management protocols. Air way was secured. IV line was secured. Vitals and oxygen saturation were monitored. Where appropriate, gastric lavage, IV fluids, inotropic support, dopamine, calcium sulphate, calcium gluconate and magnesium sulphate were given.

2.3 Data Collection \& Analysis Plan: Sex (men/ women), age groups (six), district (nine), blood pressure category and oxygen saturation category were demographic/ grouping variables (factors), while presence of mortality was a research variable. All these variables were measured on categorical scale and were analyzed by count and percentage. Estimation of parameters for the population was described by confidence interval at $80 \%$ confidence level, using an online statistical calculator "Statistics Kingdom" by normal approximation method.

\section{RESULTS}

3.1 Distribution of AIP poisoning by sex and age groups (RO 1-2): Out of a sample of 58 AIP poisoning cases and based on their confidence intervals, the frequency of AIP poisoning was higher in women $34(58.62 \%)$ than men $24(41.38 \%)$. The frequency was highest $30(51.73 \%)$ in $10-19$ years (adolescence) age group, which was steadily lower in higher age groups, and was zero in $60+$ years age group (elderly). (Table 3.1)

3.2 Distribution of AIP poisoning by district (RO 3): Most of the AIP poisoning cases were from district Swabi 17 (29.31\%), followed by district Peshawar 16 (27.58\%) cases. (Table 3.2)

3.3 Presentation of AIP poisoning by blood pressure category (RO 4): Most AIP poisoning cases $19(32.76 \%)$ presented in non-recordable blood pressure category. (Table 3.3) 
Sakhi Jan, et al.

Table 3.1: Distribution of aluminium phosphide poisoning by sex and age groups in Khyber Pakhtunkhwa Province, Pakistan $(n=58)$

\begin{tabular}{|l|l|c|c|c|c|}
\hline \multirow{2}{*}{ Variables } & \multirow{2}{*}{ Attributes } & \multicolumn{2}{|c|}{ Sample statistics } & \multicolumn{2}{c|}{$80 \%$ Cl for proportion } \\
\cline { 2 - 6 } & & Count & Percentage & Lower & Upper \\
\hline \multirow{4}{*}{ Sex } & Men & 24 & 41.38 & 33.44 & 49.79 \\
\cline { 2 - 6 } & Women & 34 & 58.62 & 50.20 & 66.55 \\
\hline \multirow{5}{*}{ Age groups } & $10-19$ years & 30 & 51.73 & 43.31 & 60.13 \\
\cline { 2 - 6 } & $20-29$ years & 18 & 31.04 & 23.24 & 38.81 \\
\cline { 2 - 6 } & $30-39$ years & 06 & 10.34 & 06.26 & 16.60 \\
\cline { 2 - 6 } & $40-49$ years & 03 & 05.17 & 02.52 & 10.28 \\
\cline { 2 - 6 } & $50-59$ years & 01 & 01.72 & 00.51 & 05.58 \\
\cline { 2 - 6 } & $60+$ years & 00 & 0 & - & - \\
\hline \multicolumn{2}{|c|}{ Total } & & 58 & $100 \%$ & \multicolumn{2}{c|}{ Population parameters } \\
\hline
\end{tabular}

Table 3.2: Distribution of aluminium phosphide poisoning by district in Khyber Pakhtunkhwa Province, Pakistan $(n=58)$

\begin{tabular}{|l|l|l|c|c|c|}
\hline \multirow{2}{*}{ Variable } & \multirow{2}{*}{ Attributes } & \multicolumn{2}{|c|}{ Sample statistics } & \multicolumn{2}{c|}{$80 \%$ Cl for proportion } \\
\cline { 2 - 6 } & & Count & Percentage & Lower & Upper \\
\hline \multirow{5}{*}{ District } & Swabi & 17 & 29.31 & 22.30 & 37.45 \\
\cline { 2 - 6 } & Peshawar & 16 & 27.58 & 20.76 & 35.64 \\
\cline { 2 - 6 } & Mardan & 07 & 12.07 & 07.60 & 18.61 \\
\cline { 2 - 6 } & Charsadda & 05 & 08.62 & 04.96 & 14.55 \\
\cline { 2 - 6 } & Buner & 04 & 06.90 & 03.71 & 12.45 \\
\cline { 2 - 6 } & Malakand & 03 & 05.17 & 02.52 & 10.28 \\
\cline { 2 - 6 } & Nowshehra & 02 & 03.45 & 01.44 & 08.01 \\
\cline { 2 - 6 } & Kohat & 02 & 03.45 & 01.44 & 08.01 \\
\cline { 2 - 6 } & Timergara & 02 & $100 \%$ & 01.44 & 08.01 \\
\hline \multicolumn{2}{|l|}{ Total } & 58 & Population parameters \\
\hline
\end{tabular}

Table 3.3: Presentation of aluminium phosphide poisoning by blood pressure category in Khyber Pakhtunkhwa Province, Pakistan $(n=58)$

\begin{tabular}{|c|l|c|c|c|c|}
\hline \multirow{2}{*}{ Variables } & \multirow{2}{*}{ Attributes } & \multicolumn{2}{|c|}{ Sample statistics } & \multicolumn{2}{c|}{$80 \%$ Cl for proportion } \\
\cline { 2 - 6 } & & Count & Percentage & Lower & Upper \\
\hline \multirow{3}{*}{$\begin{array}{c}\text { Blood Pressure } \\
\text { category (mmHg) }\end{array}$} & Non-recordable & 19 & 32.76 & 25.43 & 41.03 \\
\cline { 2 - 6 } & Below 62/42 & 06 & 10.34 & 06.26 & 16.60 \\
\cline { 2 - 6 } & $62 / 42-91 / 61$ & 13 & 22.42 & 16.21 & 30.13 \\
\cline { 2 - 6 } & $92 / 62-131 / 91$ & 14 & 24.14 & 17.71 & 31.98 \\
\cline { 2 - 6 } & Above 131/91 & 06 & 10.34 & 06.26 & 16.60 \\
\hline \multicolumn{2}{|c|}{ Total } & 58 & $100 \%$ & \multicolumn{2}{|c|}{ Population parameters } \\
\hline
\end{tabular}


3.4 Presentation of AIP poisoning by oxygen saturation category (RO 5): Most AIP poisoning cases $22(37.93 \%)$ presented in oxygen saturation category of $\geq 90 \%$. (Table 3.4 )

3.5 Prevalence of AIP poisoning mortality \& its distribution by sex and age groups (RO 6-8): Out of 58 AIP poisoning cases, 42 died, so mortality was $72.41 \%(80 \% \mathrm{Cl}, 64.35-79.23)$. The frequency of mortality was higher in women $24 / 58(41.38 \%)$ than men $18 / 58$ (31.03\%). The frequency of mortality was highest 23 (39.66\%) in 10-19 years (adolescence) age group, which was steadily lower in higher age groups. (Table 3.5)

3.6 Presentation of AIP poisoning mortality by blood pressure category (RO 9): The frequency of AIP poisoning mortality was highest as 19 (32.76\%) in non-recordable blood pressure category and was lowest as one (1.72\%) in highest blood pressure category of above 131/91. (Table 3.6)

Table 3.4: Presentation of aluminium phosphide poisoning by oxygen saturation category in Khyber Pakhtunkhwa Province, Pakistan $(n=58)$

\begin{tabular}{|c|l|c|c|c|c|}
\hline \multirow{2}{*}{ Variables } & \multirow{2}{*}{ Attributes } & \multicolumn{2}{|c|}{ Sample statistics } & \multicolumn{2}{c|}{$80 \% \mathrm{Cl}$ for proportion } \\
\cline { 2 - 6 } & & Count & Percentage & Lower & Upper \\
\hline \multirow{3}{*}{$\begin{array}{l}\text { Oxygen saturation } \\
\text { category (\%) }\end{array}$} & Below 90 & 19 & 32.76 & 25.43 & 41.03 \\
\cline { 2 - 6 } & $\geq 90$ & 22 & 37.93 & 30.20 & 46.32 \\
\cline { 2 - 6 } & Unknown & 17 & 29.31 & 22.30 & 37.45 \\
\hline \multicolumn{2}{|c|}{ Total } & 58 & $100 \%$ & \multicolumn{2}{c|}{ Population parameters } \\
\hline
\end{tabular}

Table 3.5: Distribution of AIP poisoning mortality by sex and age groups in Khyber Pakhtunkhwa Province, Pakistan $(n=58)$

\begin{tabular}{|l|l|c|c|c|c|c|}
\hline \multirow{2}{*}{ Variables } & \multirow{2}{*}{ Attributes } & \multirow{2}{*}{ Sample size } & \multicolumn{2}{|c|}{ Sample statistics } & \multicolumn{2}{c|}{$80 \%$ Cl for proportion } \\
\cline { 4 - 7 } & & & Count & Percentage & Lower & Upper \\
\hline \multirow{3}{*}{ Sex } & Men & 24 & 18 & 31.03 & 23.86 & 39.25 \\
\cline { 2 - 7 } & Women & 34 & 24 & 41.38 & 33.44 & 49.79 \\
\hline \multirow{4}{*}{ Age groups } & $10-19$ years & 30 & 23 & 39.66 & 31.81 & 48.06 \\
\cline { 2 - 7 } & $20-29$ years & 18 & 10 & 17.24 & 11.81 & 24.47 \\
\cline { 2 - 7 } & $30-39$ years & 06 & 05 & 08.62 & 04.96 & 14.55 \\
\cline { 2 - 7 } & $40-49$ years & 03 & 03 & 05.17 & 02.52 & 10.28 \\
\cline { 2 - 7 } & $50-59$ years & 01 & 01 & 01.72 & 00.51 & 05.58 \\
\hline \multicolumn{2}{|c|}{ Total deaths } & 58 & 42 & $72.41 \%$ & 64.35 & 79.23 \\
\hline \multicolumn{2}{|c|}{ No deaths } & 58 & 16 & $27.58 \%$ & 20.76 & 35.64 \\
\hline \multicolumn{2}{|c|}{ Total $(n)$} & 58 & 58 & $100 \%$ & \multicolumn{2}{l|}{ Population parameters } \\
\hline
\end{tabular}

Table 3.6: Presentation of aluminium phosphide poisoning mortality by blood pressure category in Khyber Pakhtunkhwa Province, Pakistan $(n=58)$

\begin{tabular}{|l|l|c|c|c|c|c|}
\hline \multirow{2}{*}{ Variables } & \multirow{2}{*}{ Sample size } & \multicolumn{2}{|c|}{ Sample statistics } & \multicolumn{2}{c|}{$80 \%$ Cl for proportion } \\
\cline { 3 - 6 } & Attributes & & Count & Percentage & Lower & Upper \\
\hline \multirow{4}{*}{$\begin{array}{l}\text { Blood Pressure } \\
\text { category (mmHg) }\end{array}$} & Nonrecordable & 19 & 19 & 32.76 & 25.43 & 41.03 \\
\cline { 2 - 6 } & Below 61/41 & 06 & 06 & 10.34 & 06.26 & 16.60 \\
\cline { 2 - 6 } & $62 / 42-91 / 61$ & 13 & 11 & 18.97 & 13.25 & 26.38 \\
\cline { 2 - 6 } & $92 / 62-131 / 91$ & 14 & 05 & 08.62 & 04.96 & 14.55 \\
\cline { 2 - 7 } & Above 131/91 & 06 & 01 & 01.72 & 00.51 & 05.58 \\
\hline \multicolumn{2}{|c|}{ Total deaths } & 58 & 42 & $72.41 \%$ & 64.35 & 79.23 \\
\hline \multicolumn{2}{|c|}{ No deaths } & 58 & 16 & $27.58 \%$ & 20.76 & 35.64 \\
\hline \multicolumn{2}{|c|}{ Total $(\mathrm{n})$} & 58 & 58 & $100 \%$ & \multicolumn{2}{|c|}{ Population parameters } \\
\hline
\end{tabular}


Sakhi Jan, et al.

Table 3.7: Presentation of aluminium phosphide poisoning mortality by oxygen saturation in Khyber Pakhtunkhwa Province, Pakistan $(n=58)$

\begin{tabular}{|l|l|c|c|c|c|c|}
\hline \multirow{2}{*}{ Variables } & \multirow{2}{*}{ Attributes } & \multirow{2}{*}{ Sample size } & \multicolumn{2}{|c|}{ Sample statistics } & \multicolumn{2}{|c|}{$80 \%$ Cl for proportion } \\
\cline { 3 - 6 } & & & Count & Percentage & Lower & Upper \\
\hline \multirow{3}{*}{$\begin{array}{l}\text { Oxygen saturation } \\
\text { category (\%) }\end{array}$} & Below 90 & 19 & 19 & 32.76 & 25.43 & 41.03 \\
\cline { 2 - 6 } & $\geq 90$ & 22 & 09 & 15.51 & 10.38 & 22.54 \\
\cline { 2 - 6 } & Unknown & 17 & 14 & 24.14 & 17.71 & 31.98 \\
\hline \multicolumn{2}{|c|}{ Total deaths } & 58 & 42 & $72.41 \%$ & 64.35 & 79.23 \\
\hline \multicolumn{2}{|c|}{ No deaths } & 58 & 16 & $27.58 \%$ & 20.76 & 35.64 \\
\hline \multicolumn{2}{|c|}{ Total $(\mathrm{n})$} & 58 & 58 & $100 \%$ & \multicolumn{2}{|c|}{ Population parameters } \\
\hline
\end{tabular}

3.7 Presentation of AIP poisoning mortality by oxygen saturation category (RO 10): The frequency of AIP poisoning mortality was highest 19 (32.76\%) in oxygen saturation category of below $90 \%$ and lowest $9(15.51 \%)$ in oxygen saturation category of $\geq 90 \%$. (Table 3.7).

\section{DISCUSSION}

\subsection{Distribution of AIP poisoning by sex and age groups (RO 1-2)}

Our study showed higher frequency of AIP poisoning in women $34 / 58(58.62 \%, 80 \% \mathrm{Cl}, 50.20-65.55)$ than men 24/58 (41.38\%, 80\% Cl, 33.44-49.79). (Table 3.1)

Similar to our study, higher frequency for women $59.1 \%(65 / 110)$ than men $49.9 \%(45 / 110)$ was reported by Qureshi, et al. ${ }^{23}$ from Sahiwal, Pakistan for year 2017, by Soltaninejad, et al. ${ }^{24}$ from Tehran, Iran, from 2007 to 2010 as $54.71 \%(95 \% \mathrm{Cl}, 51.55-57.83)$ (523/956) women and $45.29 \%(95 \% \mathrm{Cl}, 42.13-48.46)$ (433/956) men, by Alnaseer, et al. ${ }^{1}$ from a nationwide study from Saudi Arabia for the period from 20062017 , with $56 \%$ (38/68) women and 44\% (30/68) men, and by Louriz, et al. ${ }^{16}$ from Rabat, Morocco from January 1992-December 2007, reporting $63.27 \%$ (31/49) women and $36.73 \%$ (18/49) men.

Contrary to our study, similar frequency of AIP poisoning in men and women was reported by Iftikhar, et al. ${ }^{6}$ from Kharian, Pakistan, from July 1, 2009 to December 30, 2010, showing $25 / 52(48.1 \%, 80 \% \mathrm{Cl}$ $39.39-56.87)$ men and $27 / 52(51.9 \%, 80 \% \mathrm{Cl} 43.12-$ 60.60) women, and Shadnia, et al. ${ }^{22}$ from Tehran, Iran from January 2000 to January 2007, reporting $246 / 471(52.23 \%, 95 \% \mathrm{Cl}, 47.71-56.70)$ men and 225/471 (47.77\%, 95\% Cl, 43.29-52.28) women, and Etemadi-Aleagha, et al. ${ }^{21}$ from Tehran, Iran for the period from 2006-2013 in a toxicological/ autopsy study, reporting 967 (48.18\%) men and 1,040 $(52.82 \%)$ women. There is overlap between the two confidence intervals, hence similar.

Opposite to our study, higher frequency of AIP poisoning was shown in men 48/77 (62.34\%) than women $29 / 77$ (37.66\%) by Navabi, et al..$^{25}$ from Kermanshah province, Iran, from 2014 to 2015.

In our study, the frequency AIP poisoning was highest $30(51.73 \%)$ in $10-19$ years (adolescence) age group, followed by steady decrease as $18(31.04 \%)$ in $20-29$, six $(10.34 \%)$ in $30-39$, three $(05.17 \%)$ in $40-49$, one $(01.72 \%)$ in $50-59$ years and zero cases in $60+$ years age group (elderly). (Table 3.1)

Qureshi, et al. ${ }^{23}$ from Sahiwal, Pakistan for year 2017, reported highest frequency $48 / 110(43.64 \%)$ for age group $21-30$ years, followed by $46 / 110(41.82 \%)$ in age group $12-20,12 / 110(10.90 \%)$ in $31-40$ and $4 / 110$ $(3.64 \%)$ in age group $>40$ years.

Shadnia, et al. ${ }^{22}$ from Tehran, Iran reported highest frequency $311 / 471$ (66.02\%) for age group 21-40 years, followed by $104 / 471(22.08 \%)$ in age group $12-20,42 / 471$ (8.92\%) in $41-60$ and $14 / 471$ (2.98\%) in age group $>60$ years.

Soltaninejad, et al. ${ }^{24}$ from Tehran, Iran reported highest frequency $563 / 956$ (59\%) for age group 21 40 years, followed by $249 / 956(26 \%)$ for age group $12-20,115 / 965$ (12\%) for $41-60$ and $29 / 965$ (3\%) for age group $>60$ years.

Alnaseer, et al. ${ }^{1}$ from Saudi Arabia reported highest frequency $42 / 68(61.77 \%)$ for age group 0-20 years, followed by $19 / 68$ (27.94\%) for age group $21-40$ and $7 / 68(10.29 \%)$ for $41-60$ years age group.

It is evident from all the studies describing frequencies by age groups that as the age groups go higher, the number of cases is decreased. This might be due to the fact that youngsters are emotionally and mentally less stable as compared to adults and therefore, contribute to a higher number of self-poisonings in general.

4.2 Distribution of AIP poisoning by district (RO 3): In our study, most of the AIP poisoning cases were from district Swabi 17 (29.31\%), followed by district Peshawar 16 (27.58\%) cases. (Table 3.2)

These two areas are rich in agricultural land and thus, preservation requires the vast use of wheat pills. 
Thus, easy access of people to wheat pills in Swabi and Peshawar might contribute to the observed high number of cases. No relevant studies were available for comparison.

4.3 Presentation of AIP poisoning by blood pressure category (RO 4): In our study, most of the AIP poisoning cases $19(32.76 \%)$ were in non-recordable BP category, followed by 14 in $92 / 62-131 / 91,13$ in 62/42-91/61 and six each in $<62 / 42 \&>131 / 91$ categories. (Table 3.3)

No relevant studies were available for comparison.

4.4 Presentation of AIP poisoning by oxygen saturation category (RO 5): In our study, most of the AIP poisoning cases 22 (37.93\%) were in oxygen saturation category of $\geq 90 \%$, followed by 19 (32.76\%) in below $90 \%$ and 17 (29.31\%) in unknown category. (Table 3.4)

No relevant studies were available for comparison.

4.5 Prevalence of AIP poisoning mortality \& its distribution by sex and age groups (RO 6-8): In our study, frequency of AIP poisoning mortality was 42/58 (72.41\%, 80\%Cl, 64.35-79.23). Similar mortality to our study was reported in 1995 by Siwach, et al. ${ }^{18}$ from Haryana, India as $67.6 \%$. Lower mortality than our study was reported by Qureshi, et al..$^{23}$ from Sahiwal, Pakistan 56.36\% (62/110), Shadnia, et al. ${ }^{22}$ from Tehran, Iran 31\% (146/471), Navabi, et al. ${ }^{25}$ from Kermanshah province, Iran 53.25\% (41/77), Soltaninejad, et al. ${ }^{24}$ from Tehran, Iran, from 2007 to 2010 as $24.06 \%$ (230/956), Alnaseer, et al. ${ }^{1}$ from Saudi Arabia 32.35\% (22/68) and Louriz, et al. ${ }^{16}$ from Rabat, Morocco 49\% (24/46). Higher mortality than our study was reported by Iftikhar, et al. ${ }^{6}$ from Kharian, Pakistan 85.54\% (45/52).

In our study, AIP poisoning mortality was higher in women $24 / 58(41.38 \%, 80 \% \mathrm{Cl}, 33.44-49.79)$ than men $18 / 58(31.03 \%, 80 \% \mathrm{Cl}, 23.86-39.25)$. (Table 3.5) Contrary to our findings, similar mortality in men $117 / 956(12.24 \%)$ and women 113/956 (11.82\%) was reported by Soltaninejad, et al. ${ }^{24}$ from Tehran, Iran, and by Alnaseer, et al. ${ }^{1}$ from Saudi Arabia as 10/68 $(14.70 \%, 80 \% \mathrm{Cl}, 09.20-20.21)$ in men and $12 / 68$ $(17.65 \%, 80 \% \mathrm{Cl}, 11.72-23.57)$ in women. Opposite to our study, Shadnia, et al. ${ }^{22}$ from Tehran, Iran reported higher mortality in men 107/471 (22.72\%) than women $39 / 471$ (8.28\%).

In our study, AIP poisoning mortality was highest 23 (39.66\%) in 10-19 years (adolescence) age group, followed by steady decrease as $10(17.24 \%)$ in 20-29, five $(08.62 \%)$ in $30-39$, three $(05.17 \%)$ in $40-49$ and one (01.72\%) in 50-59 years age group. (Table 3.5) Alnaseer, et al. ${ }^{1}$ from Saudi Arabia reported highest AIP poisoning mortality $42 / 68(61.77 \%)$ in age group $0-20$ years, followed by $19 / 68$ (27.94\%) for age group $21-40$ and $7 / 68$ (10.29\%) for age group 41-60 years. Soltaninejad, et al. ${ }^{24}$ from Tehran, Iran reported highest AIP poisoning mortality 126/956 (13.18\%) for age group 21-40 years, followed by 74/956 (7.74\%) for age group 12-20, 25/965 (2.62\%) for 41-60 and $5 / 965(0.52 \%)$ for age group $>60$ years.

4.6 Presentation of AIP poisoning mortality by blood pressure category (RO 9): In our study, AIP poisoning mortality was highest $19(32.76 \%)$ in non-recordable BP category, followed by 11 (18.97\%) in 62/42-91/61, six (10.34\%) in below 61/41, five in $92 / 62-131 / 91$ and one in above 131/91 category. (Table 3.6) No relevant studies were available for comparison.

4.7 Presentation of AIP poisoning mortality by oxygen saturation category (RO 10): In our study, AIP poisoning mortality was highest $19(32.76 \%)$ in oxygen saturation category of below $90 \%$, followed by $14(24.14 \%)$ in unknown category and lowest as nine $(15.51 \%)$ in oxygen saturation category of $\geq$ 90\%. (Table 3.7) No relevant studies were available for comparison.

\section{CONCLUSIONS}

Aluminium phosphide (AIP) poisoning is an uncommon, but a dreadful public health problem in population of Khyber Pakhtunkhwa Province, Pakistan. It involves women more than men, with most cases in adolescent age group, which steadily decreases in higher age groups, with no case in $60+$ years. Most cases present with non-recordable blood pressure. AIP poisoning mortality is very high $72.41 \%$ in our population, involving more women than men, with most cases in adolescent age group, which steadily decreases in higher age groups. Mortality is highest in non-recordable blood pressure category, which steadily decreases with high blood pressure categories. Mortality is highest in oxygen saturation category of below $90 \%$.

\section{REFERENCES}

1. Alnasser S, Hussain SM, Kirdi TS, Ahmad A. Aluminum phosphide poisoning in Saudi Arabia over a nine-year period. Ann Saudi Med 2018;38(4):27783. https://doi.org/10.5144/0256-4947.2018.277

2. Wahab A, Zaheer MS, Wahab S, Khan RA. Acute aluminium phosphide poisoning: an update. Hong Kong J Emerg Med 2008;15(3):152-5. https://doi. org/10.1177/102490790801500306

3. Ghazi MA. Wheat pill (aluminum phosphide) poisoning; commonly ignored dilemma. A comprehensive clinical review. Professional Med $\mathrm{J}$ 2013;20(6):855-63. https://doi.org/10.29309/ TPMJ/2013.20.06.1829

4. Moghadamnia AA. An update on toxicology of aluminum phosphide. Daru 2012;20(1):25. https:// doi.org/10.1186/2008-2231-20-25

5. Nath NS, Bhattacharya I, Tuck AG, Schlipalius DI, Ebert PR. Mechanisms of phosphine toxicity. J Toxicol 2011;2011:494168. https://doi. org/10.1155/2011/494168

6. Iftikhar R, Tariq KM, Saeed F, Khan MB, Babar NF. 
Wheat pill: clinical characteristics and outcome. Pakistan Armed Forces Med J 2011;61(3).

7. Chugh SN, Pal R, Singh V, Seth H. Serial blood phosphine levels in acute aluminium phosphide poisoning. J Assoc Physicians India 1996 Mar;44(3):184-5.

8. Shadnia S, Rahimi M, Pajoumand A, Rasouli MH, Abdollahi M. Successful treatment of acute aluminium phosphide poisoning: possible benefit of coconut oil. Hum Exp Toxicol 2005 Apr; 24(4):2158. https://doi.org/10.1191/0960327105ht513oa

9. Sanaei-Zadeh $\mathrm{H}$, Farajidana $\mathrm{H}$. Is there a role for digoxin in the management of acute aluminum phosphide poisoning? Med Hypotheses 2011 May;76(5): 765-6. https://doi.org/10.1016/j.mehy.2011.02.023

10. Siwach SB, Singh $P$, Ahlawat S. Magnesium in aluminium phosphide poisoning--where have we erred? J Assoc Physicians India 1994;42(3):193- 4.

11. Chugh SN, Kolley T, Kakkar R, Chugh K, Sharma A. A critical evaluation of antiperoxidant effect of intravenous magnesium in acute aluminium phosphide poisoning. Magnes Res 1997 Sep;10(3):225-30.

12. Nayyar S, Nair M. Brugada pattern in toxic myocarditis due to severe aluminum phosphide poisoning. Pacing Clin Electrophysiol 2009;32(11):el6-7. https://doi.org/10.1111/j.1540-8159.2009.02522.x

13. Alter P, Grimm W, Maisch $B$. Lethal heart failure caused by aluminium phosphide poisoning. Intensive Care Med 2001;27(1):327. https://doi. org/10.1007/s001340000744

14. Mehrpour O, Jafarzadeh M, Abdollahi M. A systematic-review of aluminium phosphide poisoning. Arh Hig Rada Toksikol 2012;63(1):61-73. https://doi. org/10.2478/10004-1254-63-2012-2182

15. Tehrani H, Halvaie Z, Shadnia S, Soltaninejad K, Abdollahi M. Protective effects of Nacetylcysteine on aluminum phosphide-induced oxidative stress in acute human poisoning. Clin Toxicol (Phila) 2013 Jan;51(1):23-8. https://doi.org/10.3109/15563650 .2012 .743029

16. Louriz M, Dendane T, Abidi K, Madani N, Abouqal R, Zeggwagh AA. Prognostic factors of acute aluminum phosphide poisoning. Indian J Med Sci 2009 Jun;63(6):227-34. https://doi. org/10.4103/0019-5359.53386

17. Bogle RG, Theron P, Brooks P, Dargan PI, Red- head J. Aluminium phosphide poisoning. Emerg Med J 2006 Jan;23(1):e3. https://doi.org/10.1136/ emj.2004.015941

18. Siwach SB, Gupta A. The profile of acute poisonings in Harayana-Rohtak Study. J Assoc Physicians India 1995;43(11):756-9.

19. Singh D, Dewan I, Pandey AN, Tyagi S. Spectrum of unnatural fatalities in the Chandigarh zone of north-west India--a 25 year autopsy study from a tertiary care hospital. J Clin Forensic Med 2003 Sep;10(3):145-52. https://doi.org/10.1016/S13531131(03)00073-7

20. Gupta S, Ahlawat SK. Aluminum phosphide poisoning--a review. J Toxicol Clin Toxicol 1995;33(1):1924. https://doi.org/10.3109/15563659509020211

21. Etemadi-Aleagha A, Akhgari M, Iravani FS. Aluminium phosphide poisoning-related deaths in Tehran, Iran, 2006 to 2013. Medicine (Baltimore) 2015;94(38):e1637. https://doi.org/10.1097/ MD.0000000000001637

22. Shadnia S, Sasanian G, Allami P, Hosseini A, Ranjbar A, Amini-Shirazi N. A retrospective 7-years study of aluminum phosphide poisoning in Tehran: opportunities for prevention. Hum Exp Toxicol 2009 Apr;28(4):209-13. https://doi. org/10.1177/0960327108097194

23. Qureshi MA, Nadeem S, Ahmed T, Tariq F, Rehman $\mathrm{H}$, Qasim AP. Aluminium phosphide poisoning: clinical profile and outcome of patients admitted in a tertiary care hospital. Annals Punjab Med Coll 2018;12(3):191-4.

24. Soltaninejad K, Nelson LS, Bahreini SA, Shadnia $S$. Fatal aluminum phosphide poisoning in Tehran-Iran from 2007 to 2010. Indian J Med Sci 2012 Mar-Apr;66(3-4):66-70. https://doi. org/10.4103/0019-5359.110909

25. Navabi SM, Navabi J, Aghaei A, Shaahmadi Z, Heydari R. Mortality from aluminum phosphide poisoning in Kermanshah Province, Iran: characteristics and predictive factors. Epidemiol Health 2018 May 27;40:e2018022. https://doi.org/10.4178/epih. e2018022

CONFLICT OF INTEREST

Authors declare no conflict of interest. GRANT SUPPORT AND FINANCIAL DISCLOSURE None declared.

\section{AUTHORS' CONTRIBUTION}

The following authors have made substantial contributions to the manuscript as under:

\section{Conception or Design:}

Acquisition, Analysis or Interpretation of Data:

Manuscript Writing \& Approval:

All the authors agree to be accountable for all aspects of the work in ensuring that questions related to the accuracy or integrity of any part of the work are appropriately investigated and resolved.
SJ, HS, HN

SJ, HS, HN, AU, MB, MA

SJ, HS, HN, AU, MB, MA

Copyright (C) 2021. Sakhi Jan, et al. This is an Open Access article distributed under the terms of the Creative Commons Attribution-NonCommercial 4.0 International License, which permits unrestricted use, distribution \& reproduction in any medium provided that original work is cited properly. 